Meta

Journal des traducteurs

Translators' Journal

\title{
Modélisation du processus d'exécution des traductions
}

\section{Daniel Gouadec}

Volume 50, numéro 2, avril 2005

Processus et cheminements en traduction et interprétation

Processes and Pathways in Translation and Interpretation

URI : https://id.erudit.org/iderudit/011008ar

DOI : https://doi.org/10.7202/011008ar

Aller au sommaire du numéro

\section{Éditeur(s)}

Les Presses de l'Université de Montréal

\section{ISSN}

0026-0452 (imprimé)

1492-1421 (numérique)

Découvrir la revue

\section{Citer cet article}

Gouadec, D. (2005). Modélisation du processus d'exécution des traductions. Meta, 50(2), 643-655. https://doi.org/10.7202/011008ar

\section{Résumé de l'article}

L'auteur propose un modèle de l'exécution des prestations de traductions organisé en étapes, phases, et opérations, selon une architecture de prérequis organisant un parcours en " chemin critique " garant de la qualité. Il différencie traduction, prestation de traduction et prestation de traducteur pour intégrer la diversité des opérateurs concernés et la multiplicité des matériaux et environnements de traduction. L'objectif est de poser les cadres d'une traductologie " productive-instrumentale ». 


\title{
Modélisation du processus d'exécution des traductions
}

\author{
DANIEL GOUADEC \\ Université Rennes 2 - Haute Bretagne, Rennes, France \\ daniel.gouadec@uhb.fr
}

\begin{abstract}
RÉSUMÉ
L'auteur propose un modèle de l'exécution des prestations de traductions organisé en étapes, phases, et opérations, selon une architecture de prérequis organisant un parcours en «chemin critique» garant de la qualité. II différencie traduction, prestation de traduction et prestation de traducteur pour intégrer la diversité des opérateurs concernés et la multiplicité des matériaux et environnements de traduction. L'objectif est de poser les cadres d'une traductologie «productive-instrumentale».
\end{abstract}

\section{ABSTRACT}

An attempt to build a model of translation service provision as a sequence of steps, phases, and operations organized as an architecture of prerequisites making up the "critical path' that warrants quality. Translation proper is integrated into the wider scope of translation service provision which, in turn, is a component of the overall activity of the translator so that the wide variety of operators, materials, and translation environments is taken into account. The aim is to set up elements towards a 'productive-instrumental' perspective in translation studies.

\section{MOTS-CLÉS/KEYWORDS}

matériaux, processus, prestation, opérations, qualité

Même si les théoriciens affirment haut et clair que le traducteur doit apporter (pas nécessairement construire) ses compléments cognitifs, ses bagages spécifiques, et toutes les ressources de son art, la traduction est souvent vue comme une opération spontanée, de type quasi-épiphanique, par laquelle le traducteur procède à un décodage + transcodage + recodage instantané ou n'appelant pas la mise en œuvre de séquences particulières d'opérations. Il est généralement admis (ou implicite) que l'opération traduisante - le vocable et le singulier étant, à cet égard, particulièrement révélateurs - forme bloc et que l'essentiel du processus de traduction renvoie à la métaphore paradoxale de la boîte noire. Ainsi, les perceptions et conceptions de la traduction demeurent très largement centrées sur le texte et sur le traducteur comme début et fin de toute "chose traduisante». Elles sont majoritairement spontanéistes et syncrétiques. Et l'on peine tellement à se défaire de ces perceptions que l'on exclut du champ de la traduction tout ce qui résiste à l'enfermement dans les schémas qui fondent les perceptions et conceptions du type décrit ci-dessus. On ne s'étonne donc pas, notamment, que d'aucuns veuillent exclure la «localisation» du champ de la traduction ou cherchent à toute force à se démarquer de la doctrine implicite en parlant de transductions ou autres trans-adaptations... comme si toute forme de transfert n'était pas de la traduction. 
Le problème résulte sans doute du fait que les modèles explicites ou implicites de la traduction ne sont pas suffisamment ouverts et complets pour expliquer et analyser le traitement, par celui que l'on continue d'appeler le traducteur, d'une diversité de types de matériaux intégrés à une variété de supports et susceptibles de mobiliser, dans leur «traduction» une variété de fonctions et d'opérateurs. Il est donc sans doute opportun de s'interroger sur la modélisation du processus de traduction en décidant d'emblée que celle-ci doit s'ouvrir sur une modélisation des processus d'exécution de prestations de traducteurs qui ne se confondent pas nécessairement en tout point avec les processus d'exécution de prestations de traduction. Dans un schéma cohérent, on dirait que la prestation de traduction inclut une prestation de la part des traducteurs, qui inclut à son tour un ou des processus de traduction et que, dans un sens comme dans l'autre, ces trois niveaux sont interdépendants parce que ce qui se passe à un niveau donné détermine ce qui se passe à chacun des autres niveaux.

Il est utile de partir du constat suivant: l'activité professionnelle du personnage que l'on continue, à tort ou à raison, d'appeler traducteur recouvre une impressionnante série d'interventions que l'on peut, aux fins d'analyse et de clarification, organiser en phases, étapes, opérations, et tâches. Si l'on considère que chacune des interventions du traducteur est susceptible d'avoir une incidence sur chacune des traductions proprement dites, il apparaît légitime de commencer par dresser un tableau de ces interventions.

Nous dirons que les interventions du traducteur s'organisent en quatre phases:

a des interventions en amont/aval de toute prestation ou processus de traducteur ou de traduction [phase d'attente et prospective] ;

a des interventions en amont de l'exécution d'une prestation de traduction [phase de pré-traduction];

- des interventions liées à l'exécution d'une prestation de traduction [phase de traduction];

- des interventions en aval d'une prestation de traduction [phase de post-traduction].

Les interventions en amont et en aval de toute traduction sont toutes celles que le traducteur effectue en phase d'attente et de prospective. Elles incluent notamment, en amont des prestations de traduction: le démarchage, l'information, l'auto-formation, la formation, l'acquisition de ressources et savoir-faire, l'optimisation des ressources et savoir-faire, la définition et la promotion de l'offre de services, l'obtention de certifications. En aval des prestations de traduction, elles incluent la mise en place des savoirs, savoir-faire, ressources, et autres dérivés de chaque prestation qui vient de s'achever et mise à jour des apports de l'exécution de ladite prestation. Si ces interventions en amont et en aval se confondent, c'est tout simplement parce que le système fonctionne en boucle et que, passée la première prestation, le résultat de chaque nouvelle prestation (inter)vient en amont de la suivante.

Si nul ne considère que ces interventions relèvent du processus de traduction, il faut néanmoins reconnaître que chacune d'entre elles est de nature à influer sur les conditions particulières d'exécution de chaque prestation de traduction et donc, à l'intérieur de celle-ci, sur chaque prestation de traducteur. Ainsi, par exemple, la mise en place de mémoires de traduction et l'auto-formation du traducteur conditionnent, de toute évidence, beaucoup de choses, fût-ce parfois indirectement. 
La phase de pré-traduction inclut toutes les interventions qui, relevant de l'exécution d'une prestation de traduction (ou d'une prestation de traducteur), interviennent avant la réception du matériau à traduire, qui déclenche l'activité (et donc la phase) de traduction. Ces interventions se situent donc avant la transmission, par le "donneur d'ouvrage», du kit de traduction incluant le matériau à traiter ainsi que l'ensemble des spécifications et ressources disponibles.

Ceci signifie que la pré-traduction inclut, selon une séquence cohérente: (1) la formation du projet de traduction; (2) la sélection du ou des prestataires par le donneur d'ouvrage; (3) la formulation et la transmission de la demande de traduction par le donneur d'ouvrage; (4) l'étude de la demande et la planification prévisionnelle par le traducteur; (5) toutes les négociations entre le donneur d'ouvrage et le traducteur; (6) l'élaboration des spécifications par le donneur d'ouvrage, sous toute forme à convenir, ou la rédaction d'une proposition de prestation par le traducteur ; (7) la formation du contrat explicite ou implicite et la signature de toute convention applicable entre donneur d'ouvrage et traducteur; (8) l'acceptation définitive de la commande ou la formulation définitive du projet de traduction par le traducteur; et (9) la mise en place et la transmission du kit de traduction par le donneur d'ouvrage. La phase de pré-traduction définit ainsi l'ensemble des variables qui viendront nécessairement « encadrer», «filtrer», «contraindre» ou "déterminer » les objectifs et les procédures d'exécution de chacune des opérations prévues ou à prévoir dans la phase de traduction.

La phase de traduction démarre avec la prise en charge du kit de traduction par le traducteur. Qu'il s'agisse de traduction pure, de localisation de logiciel, de soustitrage, de localisation de site, ou autre, cette phase se subdivisera elle-même en trois étapes qui sont, dans cet ordre:

1. le pré-transfert

2. le transfert

3. le post-transfert

L'étape de pré-transfert inclut une succession d'opérations ayant pour objectif de placer le traducteur dans des conditions optimales de transfert adéquat tant en ce qui concerne l'aspect sémantique-culturel qu'en ce qui concerne l'aspect code (représentation-formulation-expression). Les opérations qui se succèdent alors en se chevauchant partiellement sont, selon une séquence très simplifiée: (1) la réception et l'analyse du kit de traduction avec relevé des carences; (2) la complémentation du kit; (3) les traitements physiques du matériau à traduire; (4) la création de la version pour traduction - qui suppose elle-même, selon les cas, le démontage ou désassemblage du matériau, le changement de support, l'extraction de séries d'éléments à traitement spécifique, le gel des balises, la désidentification, toutes les formes de conversion ou rétroconversion, et bien d'autres opérations particulières; (5) la création de la version à traduire ou retraduire effectivement; (6) la création d'une version d'appui; (7) la mise en place de l'environnement matériel et logiciel de transfert; (8) la mise en place de la version à traiter effectivement dans l'environnement de transfert; (9) la confirmation du projet ou des spécifications ; (10) la planification fine incluant, ou susceptible d'inclure, l'allotissement, l'ordonnancement, le jalonnement, les prévisionnels, le calendrier de production, la création des bons de travail; (11) le contrôle de qualité du matériau à traduire; (12) l'analyse de la version 
à traiter; (13) l'étude du produit/processus ou la documentation du sujet; (14) l'étude technique et les élucidations; (15) la mise à niveau du matériau à traduire; (16) la collecte systématique des matières premières que sont la phraséologie, la terminologie, et les modèles ou éléments antérieurement ou préalablement traduits; (17) la validation des matières premières; (18) la mise en place des matières premières dans une version d'appui; (19) les traitements anticipés et les traitements de matériaux primaires à intégrer au matériau traduit; et (20) la mise en place de la version à traiter dans l'environnement de transfert.

L'étape de transfert se définit comme le passage d'un système conceptuel-culturel et d'un système de représentation à un autre. On pourrait aussi parler de translation en ce sens que le traducteur glisse ou 'fait glisser' d'un univers à un autre. Plus prosaïquement, selon les cas, le traducteur «fait passer» le matériau à traduire «de l'autre côté» ou le traducteur passe lui-même «de l'autre côté» et, une fois passé de cet autre côté, il reconstruit ou reconstitue un matériau répondant aux impératifs et contraintes définis de cet autre côté. Il transfère ainsi, en effectuant toutes les adaptations requises, tous les éléments de contenus et de forme pertinents. Ce transfert conduit à mettre en œuvre une matière première cognitive-conceptuelle et des éléments de code de représentation (généralement, mais pas exclusivement, une matière première linguistique) dans la création d'un nouvel instrument (ouvrage, contrat, notice, etc.). Et cette mise en œuvre s'effectue dans le respect des spécifications, gabarits et procédures précédemment évoqués et dans un souci de congruence entre le matériau initial et le matériau dérivé (dérivé en ce sens qu'il résulte d'une forme d'ouvraison particulière par transformation du matériau initial). Au terme de la phase de transfert, le traducteur a mis en place, de manière plus ou moins complète et plus ou moins affirmée, un matériau que l'on dira «traduit» puisqu'il est, par définition et par opposition à du matériau produit ou conçu de manière autonome, dérivé d'un matériau préexistant, dont on a transféré, en les adaptant, les éléments pertinents de contenus et de forme. Cette phase de transfert est celle qui a reçu la plus grande attention et les processus qu'elle met en œuvre sont généralement connus, à ceci près que, dans un modèle cohérent, certains sont renvoyés à des opérations spécifiques en amont du transfert et que d'autres n'ont plus les mêmes tenants et aboutissants. L'élaboration d'un modèle doit ainsi permettre de clarifier les questions de bornage et d'intersections de phases, étapes, et opérations constitutives des processus globaux.

L'étape de post-transfert correspond à l'ensemble des opérations intervenant sur le matériau traduit afin de le rendre conforme à la fois à toutes les stéréotypies culturelles, formelles, morphologiques, techniques, linguistiques, iconographiques (ou autres) applicables dans la culture et le code destinataires ou encore selon la typologie des instruments de communication, et aux spécifications particulières définies au titre des filtres et contraintes identifiées et contrôlées lors de la phase de pré-transfert et converties en un projet de traduction [lorsque le traducteur est maître du jeu] ou, pour prendre un terme générique, en un cahier des charges [lorsque le donneur d'ouvrage commande à toute chose].

Cette étape inclut surtout l'ensemble des contrôles de qualité et mises à niveau consécutifs à la première mise en place d'un matériau traduit. Ces contrôles de qualité sont d'abord les auto-contrôles (relectures avec corrections) effectués par le traducteur. Ces auto-contrôles sont au nombre de cinq: (1) pointage du matériau traduit; 
(2) contrôle de qualité linguistique-stylistique-rédactionnelle; (3) contrôle de qualité technique-factuelle et sémantique; (4) contrôle de qualité des transferts; (5) contrôle d'homogénéité. À ces auto-contrôles peuvent bien entendu s'ajouter des contrôles externes effectués par le donneur d'ouvrage ou pour son compte et portant principalement sur la qualité technique-factuelle et sémantique et, accessoirement, pour la partie stéréotypique, sur la qualité linguistique-stylistique-rédactionnelle.

On se gardera d'oublier les contrôles de qualité fonctionnelle du matériau traduit, destinés à s'assurer que ce matériau «fonctionne» effectivement comme il le devrait et le contrôle de qualification destiné à confirmer que le matériau traduit permet effectivement à son bénéficiaire/destinataire/utilisateur d'en obtenir les effets, résultats, et fonctions escomptés ou qualité requise. Ce contrôle de qualification peut inclure un contrôle d'ergonomie destiné à confirmer que le matériau traduit n'est pas seulement fonctionnel et parfaitement exploitable mais qu'il l'est de manière optimisée en ce sens qu'il est parfaitement "adapté » à sa destination-public(s) et à sa destination-utilisation(s).

L'étape de post-transfert inclut également des opérations de finalisation du matériau traduit. La finalisation s'effectue par mises à jour, réintégration d'éléments provisoirement oblitérés, intégration d'éléments administratifs divers, et mises à niveau et mises en conformité variées.

Vient ensuite une série d'opérations que l'on situera, selon les cas, en post-transfert ou en post-traduction selon que l'on considère que le traducteur doit s'en tenir à l'exécution de la traduction ou que l'on considère au contraire que sa prestation de traducteur dépasse, parfois largement, l'exécution de la traduction au sens où elle aboutit à la mise à disposition d'un matériau traduit diffusable et donc intégré à son support fonctionnel. Les opérations envisageables sont les opérations d'intégration de premier, puis de second niveau.

L'intégration de premier niveau correspond à l'intégration du matériau traduit dans son support fonctionnel (réassemblage faisant pendant au désassemblage de l'étape de pré-transfert, plus toutes les opérations requises) assortie d'une série parfois complexe de manipulations et, bien entendu, d'une batterie de contrôles de qualité fonctionnelle et de tests de qualification/tests d'ergonomie de l'assemblage ainsi (re)constitué. Cette activité d'intégration ou de réintégration peut revêtir la forme de traitements infographiques ou de microédition, de simulation de sous-titrage, d'intégration du matériau traduit dans des menus de logiciel, d'intégration du matériau traduit dans des pages HTML du site Web, d'enregistrement de bande son, et ainsi de suite selon les supports.

L'intégration de second niveau correspond à la mise en place du premier assemblage (matériau traduit sur son support fonctionnel) sur le support ou vecteur de diffusion ou d'exploitation: création d'un exécutable, création d'un master, report de la version localisée sur la version internationale, mise en ligne du site, gravure d'un cédérom, etc.

La prestation du traducteur s'arrête lorsque la phase de post-transfert prend fin. Mais ceci ne met pas pour autant un terme à l'activité dudit traducteur. Cette activité résiduelle (mais essentielle) correspond à la phase dite de «post-traduction», qui comporte une partie que l'on dira spécifique et une partie que l'on dira générique.

La partie spécifique correspond au bouclement de la prestation achevée. Elle inclut (1) l'autopsie de la prestation qui vient de s'achever (2) le débreffage (3) l'exécution 
des opérations de facturation + recouvrement + encaissement (4) divers archivages et (5) la clôture des dossiers.

La partie générique inclut tout ce qui est susceptible de réemploi ou de remise en œuvre. Elle contribue essentiellement à une bonne préparation des traductions futures. Elle inclut, sans que la séquence soit pertinente: la consolidation des matières premières linguistiques et l'enrichissement des bases de données, avec une mention spéciale pour la mémoire de traduction, la mise à jour des outils de gestion, divers archivages et diverses opérations de suivi du matériau traduit et des partenaires. Comme nous l'avons indiqué au départ, cette partie générique vient se confondre avec la phase d'attente et de prospective.

Il apparaîtra sans doute à beaucoup que les interventions listées ci-dessus dépassent très largement le cadre de la traduction. Le critère de prise en compte est simple: si une intervention particulière ou une opération particulière a une incidence sur le déroulement ou le résultat du processus de traduction - quelles que soient la nature et la portée de ce processus - il y a lieu de l'inclure dans la modélisation de l'exécution de la traduction, de la prestation du traducteur, et de la prestation de traduction qui, comme chacun sait, mobilise d'autres opérateurs «autour du» traducteur.

Il apparaît de plus en plus clairement que l'on peut - ou même que l'on doit définir la traduction comme l'ensemble des opérations intervenant entre le moment de réception du kit de traduction et, singulièrement, du matériau à traduire et le moment où le matériau dérivé est définitivement prêt - c'est-à-dire, finalisé au sens où nous l'entendons ci-dessus, sans qu'il ait nécessairement fait l'objet d'une intégration de premier niveau ni, a fortiori, de second niveau. Si l'on considère que l'activité de traduction recouvre toutes les opérations intervenant entre ces deux moments, on comprend que l'analyse du processus de traduction doit être la somme des analyses de toutes les opérations qui entrent dans ce processus mais aussi, et peut-être d'abord, l'analyse de la manière dont ces opérations s'articulent entre elles, se suivent, se chevauchent, se recouvrent parfois, concourent ou s'excluent. Pareille analyse oblige également à s'interroger sur la nature des opérateurs concernés, sur leurs interventions respectives, et sur la manière dont ces interventions s'articulent entre elles et avec celles du traducteur.

Parler d'opérations, d'opérateurs, et de séquences d'interventions, c'est tout simplement aborder les rivages de l'analyse des chemins critiques dans un processus de transformation-ouvraison d'un matériau primaire converti en un matériau dérivé. C'est envisager le processus de traduction comme un processus de fabrication d'éléments réunis en un tout, dont la double particularité est d'être toujours une fabrication en exemplaire unique et de relever de la prestation intellectuelle.

L'analyse du processus de traduction (exécution des traductions) conduit en fait à construire autant de chemins critiques qu'il y a de configurations d'opérateurs. En effet, le chemin critique du traducteur n'est pas le même selon que le traducteur travaille en parfaite autonomie ou que le donneur d'ouvrage intervient ou qu'un chef de projet pilote ses interventions ou qu'un réviseur va intervenir, et ainsi de suite. Mais, dans tous les cas, on doit pouvoir dire, compte tenu des variables extrinsèques que sont, par exemple, les conditions de délais et de rémunération ou les spécifications du donneur d'ouvrage, quel est le parcours d'exécution optimal, pourquoi ce parcours est optimal, et pourquoi tout autre parcours présenterait 
un risque ou un inconvénient. Il reste ensuite, à l'intérieur du parcours d'exécution de la traduction, à distinguer les interventions du traducteur au cas par cas.

Raisonner ainsi en matière d'analyse du processus de traduction conduit à changer de perspective en ce sens que la traduction n'est plus nécessairement concentrée sur l'étape de transfert comme cela fut longtemps le cas parce que l'universalité apparente des référentiels pouvait laisser supposer que tout commençait et finissait avec le décodage-transcodage, mais aussi, plus tard, parce que cette perception centrée sur le transfert et la métaphore paradoxale de la boîte noire s'était enrichie d'une revendication justifiée touchant à l'importance des compléments cognitifs et contextuels-situationnels.

Nous ne parlons plus d'opération traduisante selon un vocable cher à une majorité d'analystes mais d'une séquence d'opérations constitutives d'un processus séquentiel. Nous pensons que l'on peut séparer, dissocier, distinguer des composantes souvent amalgamées afin, peut-être, d'y voir plus clair. Il s'agit donc de partir de l'idée que la traduction s'effectue par transfert construit et de traiter le processus d'exécution des traductions comme un processus séquentiel, cumulatif, décomposé en une série d'opérations elles-mêmes décomposées ou décomposables en tâches ou routines.

Il s'agit de situer l'étape de transfert entre une étape de préparation systématique et une étape de retraitement et aménagement du résultat brut du transfert et, plus largement, de confirmer que l'ensemble dépend en outre de ce qui se passe en prétraduction et de ce qui se passera en post-traduction. Le transfert n'est qu'une opération au milieu d'autres opérations, conditionnée par ces autres opérations en amont et en aval, comme le montrent de manière indiscutable l'organisation du travail mise en place dans le cadre d'exécution de projets complexes ou les dispositifs d'optimisation des contributions corrélées d'opérateurs à compétences et activités spécialisées à l'exécution de tâches standard. Bref, il s'agit de tout remettre à plat en essayant d'expliquer pourquoi, quand, et comment, les choses se font non pas spontanément, mais rationnellement.

Considérons, dans cette perspective, un segment (simplifié) du chemin critique du traducteur en pré-transfert. Ce segment concerne les interventions diverses en amont du transfert et, notamment, la collecte/réunion de la matière première. Le chemin se déroule selon la séquence ci-après :

1. Transfert du matériau à traduire sur support exploitable.

2. Numérisation ou impression, conversion ou autres traitements.

3. Démontage ou désassemblage.

4. Extractions de séries d'items à traitement spécifique (traitement anticipé; traitements particuliers).

5. Balisage/Gel des balises.

6. Suppressions ou oblitérations.

7. Repérage des segments déjà traduits.

8. Validation de (segments) de traductions existantes.

9. Repérage et compilation des sections ou segments à traduire ou retraduire effectivement.

10. Traitements de la version à traduire ou retraduire effectivement (VTRE).

11. Mise en place et tests de l'environnement de traduction.

12. Implantation de la version à traduire ou retraduire effectivement dans l'environnement de traduction. 
13. Prise en charge de la VTRE.

14. Contrôle de qualité de la VTRE sur les critères du «bon à traduire».

15. Analyse du matériau à traduire (VTRE) conduisant à la création:

- d'index des anomalies, ambiguïtés et autres défauts présumés;

- d'index pour étude du sujet, recherche documentaire, étude technique;

$\checkmark$ d'index pour la recherche linguistique (modèles, terminologie, phraséologie);

$\checkmark$ d'index des supports de traitements particuliers et/ou de traductions à options;

$\square$ notes et observations.

16. Demandes/décisions concernant les traitements particuliers et les options de traduction.

17. Demandes de ressources et d'accès aux ressources.

18. Mise à jour des directives et options.

19. Questions.

20. Élucidations directes.

21. Étude du produit ou processus concerné ou étude technique.

22. Documentation du sujet.

23. Breffage.

24. Mise à niveau du matériau à traduire (globalement ou dans la VTRE).

25. Collecte systématique des matières premières:

- Sélection et mobilisation des ressources requises;

- Compilation des répertoires, concordanciers et recueils de modèles;

- Création d'une mémoire de traduction;

- Transmission des répertoires, concordanciers, modèles et mémoires pour validation et/ou homologation.

26. Réalisation et soumission d'échantillon(s) de traduction.

27. Réception et mise en place des matières premières homologuées:

$\checkmark$ Création/mise à jour de répertoires définitifs;

- Intégration à une version d'appui existante ou à créer.

28. Réception et mise en place des directives additionnelles ou modifiées.

\section{Traitements anticipés}

Recueil de matériau de réemploi/pour intégration

(matériau primaire; éléments administratifs; autre lot de traduction)

29. Traitement de matériaux primaires, avec:

reconstitution;

compilation;

rétroconversion;

- contrôles de qualité;

u contrôle fonctionnel;

$\square$ etc.

30. Transmission pour validation ou homologation.

31. Réception et mise en place de:

$\checkmark$ résultats de traitements anticipés + confirmation des modalités de traduction;

a résultats de traitements de matériaux primaires;

32. Intégration ou montage de matériau(x) primaire(s):

$\checkmark$ dans le matériau à traduire;

$\checkmark$ dans le matériau additionnel ou lié;

33. Mise à jour de la version pour traduction effective (VTRE) ou de la version d'appui.

34. Mise en place et tests de l'environnement de transfert.

35. Intégration de la version pour transfert dans l'environnement de traduction-transfert (mise à jour). 
L'ensemble des opérations constituant l'étape de pré-transfert ci-dessus conduit à une mise en transparence absolue de l'ensemble du matériau à traduire ou retraduire effectivement. La phase antérieure de pré-traduction a précédemment permis de mettre en place un jeu de spécifications complet et affiné. L'exécution de chacune des opérations prises en compte est déterminée, encadrée, guidée, contrainte et filtrée par le jeu de spécifications applicables. Tout se passe comme si l'opérateur (le traducteur) préparait globalement le matériau à traiter effectivement, réunissait systématiquement et complètement les matières premières à mettre en œuvre, spécifiait le produit à obtenir (y compris, le cas échéant, composante par composante), puis définissait et réglait son poste de travail avant que le transfert débute avec les meilleures garanties de qualité.

Certaines opérations se décomposent elles-mêmes en séquences de sous-opérations. Ainsi, par exemple, la collecte de matériaux primaires peut s'organiser en:

$\square$ constitution d'un ou plusieurs index des matériaux primaires requis;

$\square$ identification des gisements et sources de matériaux;

$\square$ collecte des ressources;

$\checkmark$ mobilisation des outils nécessaires à l'extraction/au traitement des matériaux primaires;

$\checkmark$ extraction des matériaux primaires;

$\square$ traitements physiques requis et tests fonctionnels;

$\square$ validation (ou demande de validation);

$\square$ intégration dans toute version concernée;

$\checkmark$ contrôle d'adéquation.

Si l'on accepte la description qui précède en vertu du principe qui dit que tout ce qui peut avoir un effet positif ou négatif sur le résultat du processus (et donc sur le matériau traduit) est nécessairement une composante significative de ce processus, on peut modéliser le processus d'exécution dans sa forme la plus extensive et la plus complète en posant que toute opération obéit nécessairement au moins aux schémas, règles et contraintes suivantes:

1. identification et caractérisation du résultat escompté vu comme un élément du produit (élément de matière première ou composant du produit final) ou comme l'intégralité de ce produit (matériau traduit);

2. identification et caractérisation du matériau à traiter ;

3. identification et caractérisation de la nature du ou des traitements à appliquer ;

4. définition des moyens à mettre en œuvre en termes de:

$\checkmark$ matières premières et ressources (dont les compétences des ressources humaines/ opérateurs)

$\square$ outils et instruments requis (au moins) ou optimaux (au mieux)

$\square$ procédures;

5. identification et caractérisation des conditions de validité (et, le cas échéant, de validation) du résultat obtenu;

6. définition des remèdes à appliquer en cas de défaillance du processus.

Le modèle que l'on obtient alors apporte une description maximaliste de la prestation de traduction, de la prestation du traducteur dans les limites de la prestation de traduction, et de la phase de traduction (incluant pré-transfert, transfert et post-transfert). Il ne s'agit pas d'analyser les processus tels qu'ils interviennent ou se déroulent spontanément chez tel ou tel traducteur mais de décrire les processus tels 
qu'ils devraient se dérouler pour donner des éléments de résultat et un résultat global optimisés.

L’optique retenue n'est ni véritablement descriptive ni réellement prescriptive. Disons qu'elle est "productive-instrumentale» en ce sens qu'elle se concentre sur le processus à mettre en place et à mettre en œuvre dans tel ou tel jeu de conditions particulières. Elle repose sur le postulat que l'on peut modéliser le processus d'exécution des prestations de traduction, appliquer les modèles à l'analyse et à la conduite des processus d'exécution de la prestation du traducteur et, à l'intérieur de celle-ci, au processus de traduction. Elle permet de prendre en compte les notions primordiales de diversité de matériaux traités par le traducteur, de diversité de supports, de multiplicité d'opérateurs (partage des tâches), d'assurance de qualité (prospective) réduisant la part des contrôles de qualité, de mécanisation, et d'industrialisation.

La clé du modèle est la notion de prérequis validés au long d'un chemin dans lequel une opération ne peut avoir lieu ou produire un résultat optimal que si certaines conditions de filtrage et de mise en place d'un matériau en amont sont réunies. Construire une séquence de prérequis opération par opération, puis étape par étape, puis phase par phase, c'est concevoir l'exécution de la traduction et, au-delà, l'exécution de la prestation de traduction selon une logique de fabrication d'éléments (ou composants) combinés en un produit final (chaque produit «traduction» étant vu comme un instrument au sens global du terme). C'est aussi considérer que la gestion de l'exécution des traductions n'est rien d'autre qu'un cas particulier de la gestion de production. Et c'est enfin poser les critères du jalonnement du processus de fabrication-traduction selon la logique des chemins critiques. C'est aussi faire appel, par la force des choses, à l'ensemble des paramètres susceptibles d'exercer une influence déterminante sur la qualité des processus et de leurs résultats, puisque la pré-traduction et le pré-transfert retrouvent toute leur importance en ce sens que les prérequis sont filtrés lors de cette phase et de cette étape.

Une fois le modèle optimal construit, il reste à prendre en compte ses applications particulières en insistant sur le fait que les mises en œuvre du modèle varient selon le degré de familiarité de chacun des opérateurs (et surtout du traducteur) avec le sujet, le domaine, le type de matériau, les produits et processus du donneur d'ouvrage, la nature du donneur d'ouvrage et de ses exigences, les outils et instruments à mettre en œuvre, et toutes autres variables significatives définies au titre du projet ou des spécifications d'exécution de la tâche, des opérations ou des prestations.

Le modèle est valable partout. Il est donc unique et constant, parce qu'il repose sur une logique de prérequis et de validation des prérequis avant injection du résultat d'une opération donnée dans l'opération suivante. Ceci n'empêche en rien la variabilité des applications sur plusieurs points. Tout d'abord, dans certaines circonstances, pour tel ou tel opérateur, telle ou telle opération pourra être court-circuitée, parce que la contribution qu'elle doit apporter à l'exécution de la tâche est déjà acquise ou «en place». En second lieu, le modèle accepte les chevauchements partiels ou intégraux d'opérations critiques ou non critiques lorsque les circonstances l'exigent. Ensuite, le modèle permet de construire des boucles correspondant à des processus annexes ou secondaires de «traduction dans la traduction». Enfin, et c'est là le plus important, la mise en ouvre du modèle peut se faire de deux manières. Elle peut se faire de manière systématique, opération par opération portant sur le matériau à traiter. Elle peut aussi se faire en horizon glissant, les opérateurs avançant selon la linéarité du matériau et 
sollicitant tout prérequis en tel ou tel stade particulier du déroulement de la séquence. On peut ainsi tout aussi bien concevoir une exécution globale dans laquelle, pour simplifier, le traducteur effectue une étude systématique du produit, puis mobilise totalement la matière première terminologique pour toute sa traduction, et ainsi de suite, qu'une exécution selon la linéarité du traitement du matériau à traduire, dans laquelle le traducteur effectue des opérations ponctuelles de documentation, ou de recherche terminologique, ou autres, à chaque point de blocage du déroulement du processus - parce que, au moins pour les langues de grande diffusion, l'exploration instantanée de corpus permet la mise en transparence (corpus de même langue que le matériau à traduire) ou la mobilisation d'une matrice conceptuelle-linguistique (corpus dans la langue de traduction). Dans un cas comme dans l'autre, quoique selon des modalités différentes, le modèle dit quels sont les prérequis et explique comment et pourquoi le processus et son résultat défaillent ou risquent de défaillir lorsque ces prérequis ne sont pas mobilisés, ne sont pas mobilisés sous la forme requise, ou ne sont pas mobilisés au moment requis et donc lorsque le chemin critique n'est pas respecté.

Modéliser le processus d'exécution de la traduction, de la prestation du traducteur, et de la prestation de traduction selon les principes ci-dessus est une tâche complexe parce que le modèle doit, précisément, être universel (applicable quelles que soient les conditions de matériau, supports, opérateurs, division du travail, outils, procédures, délais, rémunération, objectifs de qualité, etc.). Mais un modèle qui ne prendrait pas toutes les variables en compte serait lui-même défaillant. La modélisation oblige d'abord à résoudre les questions de bornage et à fixer le moment du début et le moment de la fin du processus. Elle oblige aussi et surtout à prendre en compte, comme nous le faisons ici, la traduction dans la prestation du traducteur et la prestation du traducteur dans la prestation de traduction. Elle oblige donc à s'ouvrir à toutes les réalités professionnelles. Elle oblige enfin à préciser ce qui ressort à chacune des opérations et donc à chacun des domaines de savoir, de savoir-faire, et de compétences que la traduction met en jeu. Elle peut conduire à poser les fondements d'une nouvelle conception de la traduction.

La modélisation selon ces principes est en effet devenue nécessaire pour tenir compte des évolutions constatées dans les domaines et métiers de la traduction. Puisque le processus de production des traductions peut être décomposé en opérations effectuées, le cas échéant, par des opérateurs divers, l'analyse de ce même processus doit également faire l'objet d'une semblable décomposition. Ceci a le double mérite d'affiner l'analyse théorique et de permettre une optimisation des opérations et de leurs enchaînements dans la pratique professionnelle mais aussi dans la pratique pédagogique. Singulariser des opérations, c'est mettre chacune d'entre elles sous une lumière plus vive et s'obliger à prendre en compte les prérequis en amont et les implications en aval. On peut ainsi définir un chemin rationnel de la traduction entre le moment de l'arrivée du matériau à traduire et le moment de l'existence d'un matériau dérivé finalisé, tout simplement parce que l'on peut mesurer l'effet de la suppression, du déplacement ou de la modification de chacune des opérations considérées, notamment lorsque les opérations s'organisent selon un principe de chemin critique (conditions dans lesquelles une opération n'est pas possible ou ne peut avoir des résultats optimaux si elle ne s'appuie pas sur les résultats validés d'une opération antérieure). 
La modélisation ci-dessus a été effectuée dans le cadre du développement d'un logiciel de gestion de l'exécution des prestations de traduction. Elle a conduit à élaborer un modèle vu sous l'angle de chacun des opérateurs principaux, un ensemble de grilles et formulaire de recensement des variables applicables, et un jeu complet de procédures de mise en œuvre des sous-modèles. Le modèle analyse le processus d'exécution des prestations de traduction en prenant successivement comme centre de gravité et centre de l'analyse:

- le traducteur

- le donneur d'ouvrage ou le donneur d'ordres

- le courtier en traduction

- le réviseur (et tout contrôleur de la qualité) côté traducteur côté donneur d'ouvrage ou donneur d'ordres le pilote d'exécution responsable du dossier côté donneur d'ouvrage le chef de projet côté traducteur

Le résultat de la modélisation apparaît sous deux variantes d'application liées: une variante pédagogique et une variante professionnelle. Les deux modèles se rejoignent dans un même outil logiciel développé au titre du programme MLIS [projet MLIS 3010 (24928) PERFEQT, dit «ProcedurEs and Rules For Enhanced Quality in Translation»]. L'ensemble est disponible à <www.qualitrad.net $>$.

\section{RÉFÉRENCES}

\section{Ouvrages}

AFNOR (1990): La traduction, le traducteur et l'entreprise, Paris, collection AFNOR GESTION. AFNOR (1990): Terminologie - constitution des données, Paris, collection AFNOR GESTION.

GouAdec, D. (1994): Données et Informations terminologiques et terminographiques - Nature et Valeurs, Paris, Maison du dictionnaire.

Gouadec, D. (1996): Terminologie et phraséologie pour traduire - Le concordancier du traducteur, Paris, Maison du dictionnaire.

GouAdec, D. (1997): Corpus de textes et documents pour travaux pratiques de traduction et terminologie, Paris, Maison du dictionnaire.

Gouadec, D. (1999): Traduction signalétique et traduction synoptique, Paris, Maison du Dictionnaire.

GouAdec, D. (2001): Stratégies de la traduction entre l'anglais et le français - Essai de définition d'un modèle de formation des traducteurs, Métiers des langues/Langues des Métiers, Paris, Maison du Dictionnaire.

Gouadec, D. (2002): Profession: traducteur - Métiers des langues/Langues des Métiers, Paris, Maison du Dictionnaire.

Gouadec, D. (2003) : Faire traduire - Métiers des langues/Langues des Métiers, Paris, Maison du Dictionnaire.

\section{Articles}

Gouadec, D. (2000): «Procédures de l'assurance de qualité», Actes de la septième université d'été en traduction, Rennes.

Gouadec, D. (2000): Position Paper on Translator Training - Across languages and cultures I.2, p. $122-132$. 
Gouadec, D. : «Training Translators - Certainties, Uncertainties, Dilemma », in B. MaiA, J. Haller, M. UlRyCh, Training the language service provider for the new Millenium, Proceedings of the III encontros de Traducao de Astra-FLUP, p. 31-43.

Gouadec, D. (2001): «La qualité en traduction - Types et modes de traduction ", Actes de la septième université d'été en traduction, Rennes, p. 9-70.

Gouadec, D. (2001): "Concordancier terminologique - mode d'emploi », Actes de la septième université d'été en traduction, Rennes, p. 199-211.

Gouadec, D. (2002): «Professionnaliser», Actes de la huitième université d'été en traduction, Rennes, p. 217-246.

Gouadec, D. (1997): «Translator Training», Innovation and E-Learning in Translator Training (ed. A. Рyм), Tarragona, p. 11-20.

GouAdec, D. (2003) : «Formation des traducteurs: quelques grandes orientations »,La formation à la traduction professionnelle, G. Mareschal, L. Brunette, Z. Guével, E. Valentine (ed.), Presses de l'Université d'Ottawa, p. 33-45.

GouAdec, D. (2002): «Former des traducteurs: une affaire simple; un problème complexe», Actes du colloque international "territoires de la traduction", Cluj-Napoca, p. 299-314.

Gouadec, D. (2002): "Professionnaliser», Actes du colloque international sur la traduction spécialisée, Rennes, p. 217-243.

Gouadec, D. (2002): «Former de vrais professionnels», Actes du colloque international sur la traduction spécialisée Rennes, p. 245-253.

Gouadec, D. (1997): «Le bagage spécifique du localiseur/localisateur», META, 48-4, p. 526-545.

Gouadec, D. (2004) : «La révolution terminoTIC», Terminologie et Société, ed. C. de Schaetzen, p. 118-147.

Bibliographie complète à www.gouadec.net 